\title{
Stability and UV completion of the Standard Model
}

\author{
Vincenzo Branchina and Emanuele Messina ${ }^{1}$ \\ ${ }^{1}$ Department of Physics, University of Catania and INFN, \\ Sezione di Catania, Via Santa Sofia 64, I-95123 Catania, Italy
}

\begin{abstract}
The knowledge of the stability condition of the electroweak (EW) vacuum is of the greatest importance for our understanding of beyond Standard Model (BSM) physics. It is widely believed that new physics that lives at very high energy scales should have no impact on the stability analysis. This expectation has been recently challenged, but the results were controversial as new physics was given in terms of non-renormalizable higher order operators. Here we consider for the first time a renormalizable (toy) UV completion of the SM, and definitely show that such a decoupling does not take place. This result has important phenomenological consequences, providing a very useful test for BSM theories. In particular, it shows that speculations based on the so called "criticality" do not appear to be well founded.
\end{abstract}

Introduction. - The analysis of the EW vacuum stability condition is of the greatest importance for our understanding of beyond Standard Model (BSM) physics. Due to the top loop corrections, the Higgs potential $V(\phi)$ turns over for values of $\phi>v$, where $v \sim 246 \mathrm{GeV}$ is the location of the EW minimum, and develops a second minimum at a very large value $\phi_{m i n}^{(2)}$. The potential $V(\phi)$ is obtained by considering SM interactions only $1-$ [1], and depending on the Higgs and top masses, $M_{H}$ and $M_{t}$, the second minimum can be higher or lower than the EW one. When $V\left(\phi_{\min }^{(2)}\right)<V(v)$, the EW minimum is a metastable state (false vacuum), and we have to consider its lifetime $\tau$. Fig. 1 shows the usual stability phase diagram in the $M_{H}-M_{t}$ plane. The stability, instability, and metastability regions are respectively for: $V(v)<V\left(\phi_{\text {min }}^{(2)}\right) ; V\left(\phi_{\text {min }}^{(2)}\right)<V(v)$ and $\tau<T_{U}\left(T_{U}=\right.$ age of the Universe); $V\left(\phi_{\text {min }}^{(2)}\right)<V(v)$ and $\tau>T_{U}$.

When $V\left(\phi_{\text {min }}^{(2)}\right)<V(v)$, the instability scale $\phi_{\text {inst }}$ of the Higgs potential is the value of the field where $V\left(\phi_{\text {inst }}\right)=V(v)$, and $V(\phi)<V(v)$ for $\phi>\phi_{\text {inst }}$. For the present central experimental values of the Higgs and top masses, $M_{H} \sim 125.09 \mathrm{GeV}$ and $M_{t} \sim 173.34$ $\mathrm{GeV}$ [12, 13, it turns out that $\phi_{\text {inst }} \sim 10^{11} \mathrm{GeV}>>v$, $\phi_{\text {min }}^{(2)} \sim 10^{30} \mathrm{GeV}$, and $\tau$ is much larger than $T_{U}$. Naturally, new physics interactions are expected to have an effect long before the scale $\phi_{\text {min }}^{(2)} \sim 10^{30} \mathrm{GeV}$ is reached. The analysis outlined above is done under the assumption that new physics shows up only at very high energy scales, possibly the Planck scale. Moreover, it is assumed that, despite the presence of these new physics interactions, $\tau$ can be calculated with the potential obtained with SM interactions only [8, 14]. In fact, it is argued that the relevant scale for tunneling is the instability scale $\phi_{\text {inst }} \sim 10^{11} \mathrm{GeV}$, and that the contribution to the tunneling rate coming from very high scale physics ( $>>\phi_{\text {inst }}$ ) should be suppressed (decoupling) 14].

Contrary to these expectations, there are indications 15 17. that the presence of new physics at high energy scales can strongly modify the stability analysis. In refs. [15 17, however, new physics interactions are parametrized in terms of few higher order, nonrenormalizable operators. For this reason, these results are considered with a certain skepticism, and it is suggested that when the infinite tower of higher dimensional (new physics) operators of the renormalizable UV completion of the SM is taken into account, the effect should disappear, and the expected decoupling should be recovered. Actually, it is thought that this effect takes place above the physical cutoff, where the control of the theory is lost 18.

In this Letter we consider for the first time a fully renormalizable (toy) UV completion of the SM, where new physics interactions live at scales much higher than the instability scale $\phi_{\text {inst }}$, and perform the stability analysis of the EW vacuum. We shall then be able to provide a definite answer to the crucial question of whether the stability condition of the EW vacuum is affected by the presence of very high scale new physics or, as commonly expected, a decoupling takes place 14]. A clear understanding of this issue is of the greatest importance for BSM physics, provides very useful guidance for BSM model building, and is the main motivation for the present work.

The model. - The classical potential for the Higgs doublet

$$
\Phi=\frac{1}{\sqrt{2}}\left(\begin{array}{c}
-i\left(G_{1}-i G_{2}\right) \\
\phi+i G_{3}
\end{array}\right)
$$

where $\phi$ is the Higgs field and $G_{i}$ are the Goldstones, is:

$$
U(\Phi)=m^{2}\left(\Phi^{\dagger} \cdot \Phi\right)+\lambda\left(\Phi^{\dagger} \cdot \Phi\right)^{2} .
$$

Our (toy) renormalizable UV completion of the SM is obtained by considering the addition of a scalar field $S$ and a fermion field $\psi$ that interact in a simple way with $\Phi$, and have masses $M_{S}$ and $M_{f}$ well above the instability scale, $M_{S}, M_{f}>>\phi_{\text {inst }}$. Apart from the kinetic terms, the additional terms in the Lagrangian are:

$$
\begin{aligned}
\Delta \mathcal{L} & =\frac{M_{S}^{2}}{2} S^{2}+\frac{\lambda_{S}}{4} S^{4}+2 g_{S}\left(\Phi^{\dagger} \cdot \Phi\right) S^{2} \\
& +M_{f}\left(\bar{\psi}_{L} \psi_{R}+\bar{\psi}_{R} \psi_{L}\right) \\
& +\sqrt{2} g_{f}\left(\bar{\Psi}_{L} \cdot \Phi \psi_{R}+\bar{\psi}_{R} \Phi^{\dagger} \cdot \Psi_{L}\right)
\end{aligned}
$$




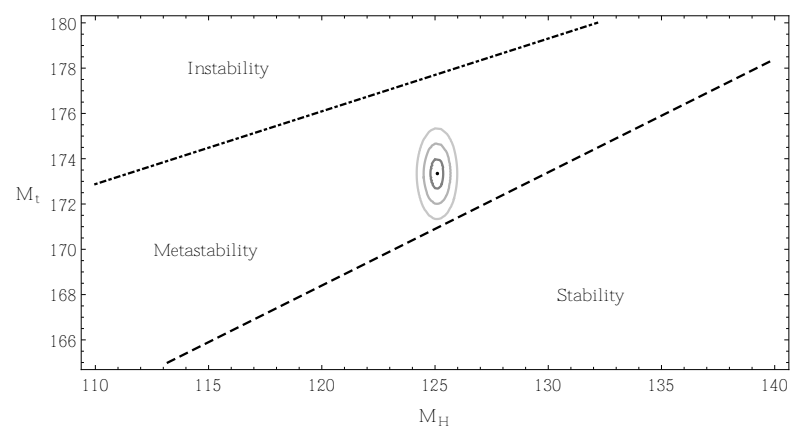

FIG. 1: This figure shows the usual stability diagram for the EW vacuum, done under the assumption that new physics interactions at very high scales have no impact on its stability condition [8] 10] The $M_{H}-M_{t}$ plane is divided in three sectors (see text): absolute stability, metastability and instability regions. The dot indicates the current central experimental values $M_{H} \sim 125.09 \mathrm{GeV}$ and $M_{t} \sim 173.34 \mathrm{GeV}$, and the corresponding EW vacuum lifetime is $\tau \sim 10^{600} T_{U}$ (see text).

where $\lambda_{S}$ is the self-coupling of the new scalar $S, g_{S}$ the coupling between the Higgs doublet and $S, \psi_{L}$ and $\psi_{R}$ the left and right components $(S U(2)$ singlets) of the Dirac field $\psi$ with mass $M_{f}, \Psi_{L}$ the left-handed $S U(2)$ fermion doublet $\Psi_{L}=\left(0, \psi_{L}\right)^{T}$ (we are not considering additional neutrinos), and $g_{f}$ the Yukawa coupling between $\Psi$ and the Higgs doublet $\Phi$.

Inserting (1) in (3) we have:

$$
\begin{aligned}
\Delta \mathcal{L} & =\frac{M_{S}^{2}}{2} S^{2}+\frac{\lambda_{S}}{4} S^{4}+g_{S} \varphi^{2} S^{2}+M_{f} \bar{\psi} \psi \\
& +g_{f} \varphi \bar{\psi} \psi+g_{S}\left(G_{1}^{2}+G_{2}^{2}+G_{3}^{2}\right) S^{2} \\
& +g_{f} G_{3} \bar{\psi}\left[\left(\frac{1+\gamma_{5}}{2}\right)+i\left(\frac{1-\gamma_{5}}{2}\right)\right] \psi
\end{aligned}
$$

For the purposes of the present work, it is sufficient to consider the impact of these additional terms on the Higgs potential $V(\phi)$ at the one-loop level only. Then, in the following we do not need to consider further the second and the third lines of Eq. 44. The one-loop contribution to $V(\phi)$ from the additional terms in $\Delta \mathcal{L}$ is:

$$
\begin{aligned}
V_{1}(\phi) & =\frac{\left(M_{S}^{2}+2 g_{S} \phi^{2}\right)^{2}}{64 \pi^{2}}\left[\ln \left(\frac{M_{S}^{2}+2 g_{S} \phi^{2}}{M_{S}^{2}}\right)-\frac{3}{2}\right] \\
& -\frac{\left(M_{f}^{2}+g_{f}^{2} \phi^{2}\right)^{2}}{16 \pi^{2}}\left[\ln \left(\frac{M_{f}^{2}+g_{f}^{2} \phi^{2}}{M_{S}^{2}}\right)-\frac{3}{2}\right]
\end{aligned}
$$

where the renormalization scale is chosen at $\mu=M_{S}$.

According to the decoupling argument [8, 14], these new physics interactions at very high energy scales $\left(M_{S}, M_{f}>>\phi_{\text {inst }} \sim 10^{11} \mathrm{GeV}\right)$ should have no impact on the stability analysis. We now investigate this question by considering two choices for the parameters of our (toy) UV completion of the SM. In both cases, a second minimum deeper than the EW one is formed, and we then have to calculate the EW vacuum lifetime.

Results. - We now impose to the modified potential $V(\phi)=\frac{\lambda}{4} \phi^{4}+V_{1}(\phi)$ (as usual the quadratic term can be neglected as we consider very high values of $\phi)$ the matching conditions at the threshold scale $M_{f}$ so that the SM Higgs potential is recovered for values of $\phi<M_{f}$. The EW vacuum lifetime $\tau$ is then given by [17]

$$
\tau=\min _{\mu}\left(\frac{1}{T_{U}^{3} \mu^{4}} \exp \frac{8 \pi^{2}}{\left(3\left|\lambda_{S M}(\mu)+4 \bar{V}_{1}(\mu) / \mu^{4}\right|\right)}\right)
$$

where $\lambda_{S M}(\mu)$ is the running quartic coupling, and $\bar{V}_{1}(\phi)$ is nothing but the additional contribution (5) to the Higgs potential with the $\phi^{2}$ and $\phi^{4}$ terms subtracted.

Before going on with the calculation of $\tau$ for our model, it is worth to remind that when the presence of these new physics interactions (usually assumed to be harmless for calculating $\tau$ ) is neglected, for the present central values of the Higgs and top masses, $M_{H}=125.09 \mathrm{GeV}$ and $M_{t}=173.34 \mathrm{GeV}$ [12, 13, the calculation gives:

$$
\tau \sim 10^{600} T_{U}
$$

This result is the basis for the so called metastability scenario, according to which although the EW minimum is a metastable state (and then a false vacuum), as its lifetime turns out to be much larger than the age of the universe, we may well live in such a state.

Fig.1 shows the full stability analysis done under the assumption that new physics at scales $>>\phi_{\text {inst }}$ has no impact on the stability condition of the EW vacuum [811. The black dot corresponds to the tunneling time of Eq. (7). The ellipses give the one, two and three sigma experimental uncertainties.

We move now to the computation of the EW vacuum lifetime for our model with new physics at very high energy scales $\left(>>\phi_{\text {inst }}\right)$. For our first example, we choose $M_{S}=1.2 \cdot 10^{18} \mathrm{GeV}, M_{f}=0.6 \cdot 10^{17} \mathrm{GeV}$, $g_{S}\left(M_{S}\right)=0.97, g_{f}^{2}\left(M_{S}\right)=0.48, \lambda_{S M}\left(M_{S}\right)=-0.0151$. The latter is the value of the running quartic coupling $\lambda_{S M}(\mu)$ at the scale $\mu=M_{S}$, obtained by considering the RG equations for the SM coupling constants and the boundary conditions at the next-to-next to leading order [10, 19]. Note that at the order of approximation that we are considering, $\lambda_{S}$ plays no role.

For the values of the parameters given above, the Higgs effective potential $V(\phi)$ develops a new minimum, lower than the EW one, at $\phi_{\text {min }} \sim 0.4 \cdot 10^{19} \mathrm{GeV}$. To study the stability condition of the EW vacuum, we have then to calculate its lifetime $\tau$. For the present central experimental values of the Higgs and top masses $\left(M_{H}=125.09\right.$ $\mathrm{GeV}$ and $M_{t}=173.34 \mathrm{GeV}$ ) we find:

$$
\tau \sim 10^{180} T_{U}
$$

This result has to be compared with the tunneling time of Eq. (7), obtained by considering the SM potential alone (no new physics included). Although for this example the tunneling time is still much higher than the age of the Universe, Eq. (8) gives a result that is greatly different from the one of Eq. (7). 


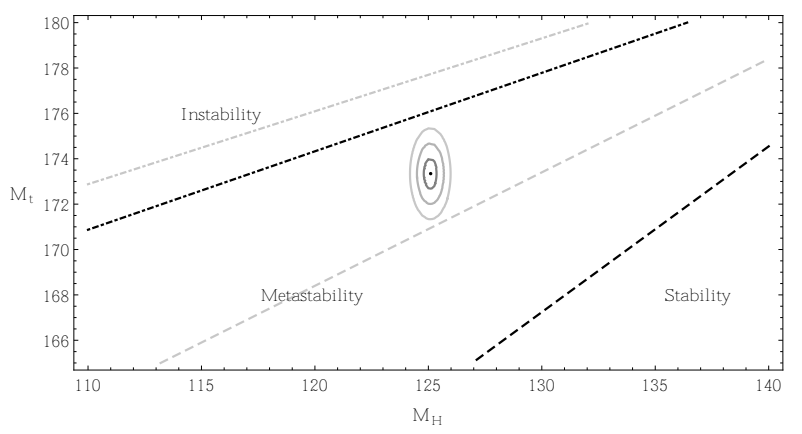

FIG. 2: This figure shows the stability diagram for the EW vacuum in the $M_{H}-M_{t}$ plane when the toy (very high energy) UV completion of the SM given in Eq. (3) is considered. The Higgs effective potential is modified by the presence of the additional term $(5)$. Here $M_{S}=1.2 \cdot 10^{18} \mathrm{GeV}, M_{f}=0.6 \cdot 10^{17}$ $\mathrm{GeV}, g_{S}=0.97, g_{f}^{2}=0.48$. As in fig. 1 , the $M_{H}-M_{t}$ plane is divided in three sectors: absolute stability, metastability and instability regions. The presence of the term (5) causes the lowering of the instability and stability lines.

Let us consider now another example, with a different value of $M_{f}$. More precisely, let us take $M_{S}=1.2 \cdot 10^{18}$ $\mathrm{GeV}, M_{f}=2.4 \cdot 10^{15} \mathrm{GeV}, g_{S}\left(M_{S}\right)=0.97, g_{f}^{2}\left(M_{S}\right)=$ 0.48 , and $\lambda_{S M}\left(M_{S}\right)=-0.0151$. Again for the present central experimental values of $M_{H}$ and $M_{t}$ we find:

$$
\tau \sim 10^{-65} T_{U}
$$

In this case, the situation is more dramatic than in the previous example: the tunneling time turns out to be much smaller than the age of the Universe. If realistic, this model could not be accepted.

The lesson from Eqs. (7), (8), and (9) is clear. The expectation that the tunneling time should be insensitive to physics that lives at energies higher than the instability scale, in other words that the result shown in Eq. (7) should not be modified by the presence of new physics at high energies, is not fulfilled.

Naturally we may ask why the decoupling argument fails. The reason is that the decoupling theorem applies when we calculate scattering amplitudes at energies $E$ lower than $M_{S}$ and $M_{f}$. In these cases, the contributions from high energy new physics is suppressed by factors as $E / M_{S}$ and $E / M_{f}$ to some appropriate power.

In our case, however, we are calculating the tunneling time. Tunneling is a non-perturbative phenomenon, and no decoupling applies: in the calculation of $\tau$, no naive suppression factor, $\phi_{\text {inst }} / M_{S}$ or $\phi_{\text {inst }} / M_{f}$, appears. More technically, the tunneling time $\tau$ is essentially given by the exponential in Eq. (6), whose argument is the action calculated at bounce solution to the euclidean EulerLagrange equation of motion [20. If the Higgs potential is modified by the presence of terms as the one in Eq. (5), the new bounce turns out to be different from the one obtained when this term is absent. The action is modified and (once exponentiated) gives rise to a value for $\tau$ that can be enormously different from the result obtained when new physics is not considered.

This is a central result of the present work. With the help of a fully renormalizable (toy) UV completion of the SM, we have firmly shown that, contrary to a widely diffused expectation, the EW vacuum lifetime strongly depends on new physics even if the latter lives at very high energy scales, much higher than the instability scale $\phi_{\text {inst }} \sim 10^{11} \mathrm{GeV}$. As we have just shown, this phenomenon is not due to an illegitimate extrapolation of the theory beyond its validity [18].

On the contrary, it is an illegitimate application of the decoupling argument to a phenomenon to which it cannot be applied, namely the (non-perturbative) tunneling phenomenon, that leads to the expectation that physics at scales much higher than the instability scale $\phi_{\text {inst }}$ should have no impact on the stability condition.

We are now ready to proceed with our analysis. Fig.1 shows the stability diagram in the $M_{H}-M_{t}$ plane obtained under the assumption (decoupling argument) that the stability analysis does not depend on high energy physics. The examples considered above, with the results (7), (8) and (9), indicate that we should expect that the whole stability phase diagram actually depends on new physics, even if it lives at very high energy scales.

The dashed and the dashed-dotted lines of fig. 1 are named the stability line and the instability line respectively. The first one is obtained for those values of $M_{H}$ and $M_{t}$ such that the two minima are at the same height, the latter is obtained for the case when $V\left(\phi_{\min }^{(2)}\right)<V(v)$ and $\tau=T_{U}$.

Let us repeat now the stability analysis when the term (3), that is our (toy) UV completion of the SM, is added to the SM Lagrangian, so that the term (5) is added to the Higgs effective potential. In fig. 2, the analysis is performed for the values of the parameters considered in our first example, namely $M_{S}=1.2 \cdot 10^{18} \mathrm{GeV}$, $M_{f}=0.6 \cdot 10^{17} \mathrm{GeV}, g_{S}\left(M_{S}\right)=0.97, g_{f}^{2}\left(M_{S}\right)=0.48$, $\lambda_{S M}\left(M_{S}\right)=-0.0151$.

We note that the instability line moves downwards. This result had to be expected from the previous results (7) and (8) for the tunneling time. In fact, we obtained $\tau \sim 10^{18} T_{U}$ for the UV completed Higgs potential and $\tau \sim 10^{600} T_{U}$ for the SM Higgs potential. It is clear that in the case of the UV completed potential, the experimental point (black dot) must be closer to the instability line than in the case of the unmodified potential. The grey lines of fig. 2 are the old instability and stability lines for the the unmodified Higgs potential (see fig. 1).

Actually, another important effect is that even the stability line moves downwards (see fig. 2). When it was thought that a decoupling effect assured that new physics at high scales could not modify this diagram, many speculations were triggered by the fact that the experimental point (black dot in the figure) $M_{H} \sim 125.09 \mathrm{GeV}$ and $M_{t} \sim 173.34 \mathrm{GeV}$ lies "close", within 2-3 sigma, to the stability line.

In this respect, it was suggested that more refined measurements of $M_{t}$ and $M_{H}$ should allow to determine 


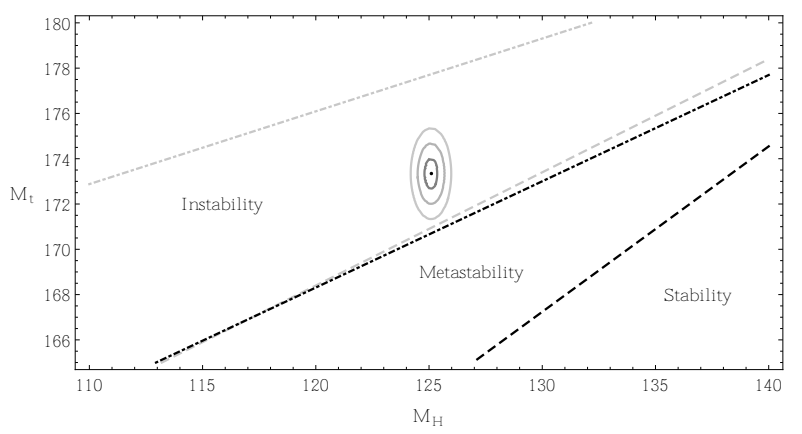

FIG. 3: The same as in fig. 2, for different values of the parameters. Here $M_{S}=1.2 \cdot 10^{18} \mathrm{GeV}, M_{f}=2.4 \cdot 10^{15} \mathrm{GeV}$, $g_{S}=0.97, g_{f}^{2}=0.48$. As in fig. 2 , the presence of the term (5) causes the lowering of the instability and the stability lines. However, in this case the instability line goes below the experimental point, signaling that the model, for these values of the parameters, cannot be considered as a viable UV completion of the SM.

whether the $\mathrm{EW}$ vacuum is a stable or a metastable state. Some authors even went to the point to consider the closeness of the experimental point to the stability line as the most important message from LHC [11], speculating on this closeness and elaborating on it for model building [18].

The results presented in this letter show that the stability condition of the EW vacuum is much more sensitive to high energy new physics than to the values of the Higgs and top masses. Therefore, more refined measurements of $M_{t}$ and $M_{H}$, that are clearly very important on their own, will not allow to determine the stability condition of the EW vacuum.

Moreover, speculations and model building inspired by the so called "criticality condition", the closeness of the experimental point to the stability (also called critical) line, are actually unjustified. As we have seen, new physics even if it lives at very high energies (we certainly expect new physics at least at very high energies, maybe Planck scale) can enormously modify the distance between the experimental point and the critical line.

Finally, in fig. 3 the stability diagram for our model with the values of the parameters considered in our second example $\left(M_{S}=1.2 \cdot 10^{18} \mathrm{GeV}, M_{f}=2.4 \cdot 10^{15} \mathrm{GeV}\right.$, $\left.g_{S}\left(M_{S}\right)=0.97, g_{f}^{2}\left(M_{S}\right)=0.48, \lambda_{S M}\left(M_{S}\right)=-0.0151\right)$ is shown. The instability and stability lines move downwards as for the previous case. In this case, however, the tunneling time for the experimental point is much shorter than the age of the Universe, see Eq. (9), and in fact we see that the experimental point is now inside the instability region.

This simply means that the model with these values of the parameters cannot be considered as a viable UV completion of the SM. This result contains another important lesson of the present work. The stability condition of the EW vacuum, as we have shown, is strongly sensitive to new physics, even when it lives at very high energy scales. Therefore, as we have shown that for the stability analysis we cannot rely on the decoupling of high energy physics, we conclude that candidate BSM theories have to be checked against a sort of stability test. Only models with a stable or metastable (but with $\tau>T_{U}$ ) EW vacuum can be considered as viable UV completions of the SM.

Conclusions. - With the help of a (toy) renormalizable UV completion of the SM, we have definitely shown that new physics interactions, even when they live at energies much higher than the scale where the Higgs potential becomes unstable (the so called instability scale $\phi_{\text {inst }} \sim 10^{11} \mathrm{GeV}$ ), have strong impact on the stability condition of the EW vacuum. With respect to previous analyses, here new physics interactions are given in terms of a fully renormalizable theory rather than with the help of higher order non-renormalizable operators, and this makes the conclusions of the present work really robust. These results have far reaching phenomenological consequences, providing very useful guidance for BSM model building. In particular, they show that speculations based on the so called "criticality" are not well founded.

[1] N. Cabibbo, L. Maiani, G. Parisi, R. Petronzio, Nucl. Phys. B158 (1979) 295.

[2] R.A. Flores, M. Sher, Phys. Rev. D27 (1983) 1679; M. Lindner, Z. Phys. 31 (1986) 295; M. Sher, Phys. Rep. 179 (1989) 273; M. Lindner, M. Sher, H. W. Zaglauer, Phys. Lett. B228 (1989) 139.

[3] C. Ford, D.R.T. Jones, P.W. Stephenson, M.B. Einhorn, Nucl. Phys. B395 (1993) 17.

[4] M. Sher, Phys. Lett. B317 (1993) 159.

[5] G. Altarelli, G. Isidori, Phys. Lett. B337 (1994) 141.

[6] J.A. Casas, J.R. Espinosa, M. Quirós, Phys. Lett. B342 (1995) 171; Phys. Lett. B382 (1996) 374.

[7] F. Bezrukov, M. Yu. Kalmykov, B. A. Kniehl, M. Shaposhnikov, JHEP 1210 (2012) 140.

[8] G. Isidori, G. Ridolfi, A. Strumia, Nucl. Phys. B609 (2001) 387.

[9] J. Elias-Miro, J.R. Espinosa, G.F. Giudice, G. Isidori, A. Riotto, A. Strumia, Phys. Lett. B709 (2012) 222.

[10] G. Degrassi, S. Di Vita, J. Elias-Miro, J.R. Espinosa, G.F. Giudice, G. Isidori, A. Strumia, JHEP 1208 (2012) 098.

[11] D. Buttazzo, G. Degrassi, P. P. Giardino, G. F. Giudice, F. Sala, A. Salvio, A. Strumia, JHEP 1312 (2013) 089.

[12] G. Aad et al. (ATLAS Collaboration, CMS Collaboration), Phys. Rev. Lett. 114 (2015) 19.

[13] ATLAS and CDF and CMS and D0 Collaborations, "First combination of Tevatron and LHC measurements of the top-quark mass," arXiv:1403.4427 [hep-ex].

[14] J. R. Espinosa, G. F. Giudice and A. Riotto. JCAP 0805, 002 (2008), arXiv:0710.2484 [hep-ph].

[15] V. Branchina and E. Messina, Phys. Rev. Lett. 111 (2013) 241801, arXiv:1307.5193 [hep-ph].

[16] V. Branchina, E. Messina and A. Platania, JHEP 1409 (2014) 182, arXiv:1407.4112 [hep-ph].

[17] V. Branchina, E. Messina and M. Sher, Phys.Rev. D91 
(2015) 1, 013003, arXiv:1408.5302 [hep-ph].

[18] J. R. Espinosa, G. F. Giudice, E. Morgante, A. Riotto, L. Senatore, A. Strumia and N. Tetradis, arXiv:1505.04825 [hep-ph].

[19] L.N. Mihaila, J. Salomon and M. Steinhauser, Phys. Rev. Lett. 108 (2012) 151602; K. Chetyrkin and M. Zoller,
JHEP 06 (2012) 033; F. Bezrukov, M. Yu. Kalmykov, B. A. Kniehl, M. Shaposhnikov, JHEP 1210 (2012) 140.

[20] S. Coleman, Phys. Rev. D15 (1977) 2929; C. G. Callan, S. Coleman, Phys. Rev. D16 (1977) 1762. 